\title{
Metabolic Acidosis in the Surgical Intensive Care Unit: Risk Factors, Clinical Correlates and Outcome. Findings from a High Dependency Heart and Vascular Surgical Center in Nigeria
}

\author{
P. K. Uduagbamen ${ }^{*}{ }^{(0)}$, M. Sanusi'2, O. B. Udom², O. F. Salami' ${ }^{3}$ A. D. Adebajo², O. J. Alao1 \\ ${ }^{1}$ Division of Nephrology and Hypertension, Department of Internal Medicine, Ben Carson (Snr) School of Medicine, Babcock \\ University/Babcock University Teaching Hospital, Ilishan-Remo, Nigeria \\ ${ }^{2}$ Tristate Heart and Vascular Center, Babcock University Teaching Hospital, Ilishan-Remo, Nigeria \\ ${ }^{3}$ Intensive Care Unit, Department of Surgery, Ben Carson (Snr) School of Medicine, Babcock University/Babcock University \\ Teaching Hospital, Ilishan-Remo, Nigeria \\ Email: *petr.uduagbamen@gmail.com
}

How to cite this paper: Uduagbamen, P.K., Sanusi, M., Udom, O.B., Salami, O.F., Adebajo, A.D. and Alao, O.J. (2020) Metabolic Acidosis in the Surgical Intensive Care Unit: Risk Factors, Clinical Correlates and Outcome. Findings from a High Dependency Heart and Vascular Surgical Center in Nigeria. World Journal of Cardiovascular Surgery, 10, 226-241.

https://doi.org/10.4236/wjcs.2020.1011025

Received: September 14, 2020

Accepted: November 27, 2020

Published: November 30, 2020

Copyright ( 2020 by author(s) and Scientific Research Publishing Inc. This work is licensed under the Creative Commons Attribution International License (CC BY 4.0).

http://creativecommons.org/licenses/by/4.0/ (c) (i) Open Access

\begin{abstract}
Background: Metabolic acidosis (MA) is a common finding on the surgical ward, more so in the intensive care unit. Diseases affecting the major organ systems of the body and higher grades of surgery are common risk factors for MA. It is associated with poor treatment outcome. Aim: To determine the risk factors and clinical correlates of metabolic acidosis and assess its relationship with treatment outcome. Methodology: A retrospective study at the "Tristate Heart and vascular Center" in Ilishan-Remo South west Nigeria, on patients that had cardiac and vascular surgeries from January 2015 to December 2019. Three hundred and forty two participants took part in the study. The demographic, clinical and laboratory findings were entered. Statistical analysis was with Student's t-test and Chi square. Results: Two hundred and six males and 136 females were studied. The incidences of metabolic acidosis prior to induction, on post-operative day one $\left(\mathrm{POD}^{1}\right)$ and on $\mathrm{POD}^{28}$ were $20.7 \%, 39.8 \%$ and $14.1 \%$ respectively. Nine (2.6\%) participants died during admission, of this, 6 (66.7\%) had MA at presentation but all (100\%) had $\mathrm{MA}$ on $\mathrm{POD}^{1}$. The Risk factors for MA were advanced age, comorbidities, open heart surgery, elevated systolic blood pressure and low eGFR. Metabolic acidosis was a risk factor for prolonged hospital stay, perioperative death and declining kidney function which was commoner among participants with preexisting kidney dysfunction. Conclusion: The incidence of metabolic acidosis was $20.7 \%$ at induction of anesthesia, rose to $39.8 \%$ on $\mathrm{POD}^{1}$ and by $\mathrm{POD}^{28}$, it has significantly reduced to $14.1 \%$. While advancing age and com-
\end{abstract}


orbidities were risk factors for MA, the occurrence of MA increased the risk of declining kidney function, prolonged hospital stay and death.

\section{Keywords}

Metabolic Acidosis, Perioperative, Anesthetic Induction, Post-Operative Day, Open Heart, Vascular, Comorbidities, Kidney Function

\section{Introduction}

Metabolic acidosis (MA), defined as elevated hydrogen ions $\left(\mathrm{H}^{+}\right)$concentration in the blood, is a common finding in most illnesses, particularly those affecting the major organ systems of the body [1]. It is reported to be a risk factor for poor treatment outcome in many medical and surgical conditions, more so in highly dependent intensive care units dedicated for managing patients with advance cardiac and vascular procedures, due to greater incidence of perioperative comorbidities and complications [2]. The body in normal state balances the net acid production (NAP) with the net acid excretion (NAE) through tubular generation of ammonium salt $\left(\mathrm{NH}_{4}\right)$ and excretion of an acidic urine [3]. Metabolic acidosis would therefore result from net acid over production, reduced $\mathrm{NH}_{4}$ generation and subsequent low NAE or in primary dysfunctional tubular $\mathrm{NH}_{4}$ generation. The activation of the renin angiotensin aldosterone pathway (RAAS), with the sympathetic nervous system inhibits the renal excretion of $\left(\mathrm{H}^{+}\right)$ions that occurs through the activation of the atrial natriuretic peptides (ANP) induced by increased atrial pressures [4]. Angiotensin II is also a potent stimulator of the NADPH oxidase for the release of reactive oxygen species, cytokines and hydrogen ions with resultant lowering of the blood $\mathrm{PH}$ [5].

Metabolic acidosis is most commonly assessed by determining the serum bicarbonate $\left(\mathrm{HCO}_{3}^{2-}\right)$ level either by the total carbon dioxide concentration or by arterial blood gases $(\mathrm{ABG})$ using the Henderson-Hasselbach equation with the $\mathrm{PH}$ of blood and the partial pressure of carbon dioxide [6]. A good correlation is reported between these measures while another study reported wide differences in findings from the two methods [7] [8]. The differences could however, be as a result of the differences in timing and in sample compartments. [8] Locally analyzed samples are reported to give lower bicarbonate values compared with pooled samples assessed in large central laboratories due to gas leakage that results from changes in atmospheric pressure during flights, in addition to sample exposure in larger carriage tubes [9] [10].

The incidence of preoperative MA is reported to be $17 \%$ [11]. Metabolic acidosis arising from acute inflammatory conditions presents with worse prognosis in males whereas in chronic inflammatory conditions, females fare worse. The ability of females to mount greater inflammatory response, with higher erythrocyte sedimentation rate (ESR), C-reactive protein and neutrophil count, in acute settings, most likely accounts for the better outcome for females. However, in 
chronic inflammatory states, with the exception of connective tissue disease, males have less morbidity and mortality [12].

Aging is associated with lowering of serum bicarbonate which is reported to be directly related to the glomerular filtration rate (GFR) in health and in chronic kidney disease (CKD) [13]. Individuals with cardiac and vascular diseases needing surgical corrections commonly present with diminished GFR, either from relative renal hypoperfusion as a result of reduced cardiac output, or as part of a wider spectrum of diseases of the organ systems particularly the kidneys, heart and the brain. The aging process is commonly associated with poor urine concentration, reduced total body water (TBW) and reduced excretion of water, sodium and potassium [14]. The elderly commonly have elevated antidiuretic hormone coupled with massively elevated atrial natriuretic peptide hence the higher prevalence of hyponatremia in them [15].

Aging is associated with reductions in kidney sizes and function, with a relatively greater affectation of the renal cortex than the medulla [16]. Between 30 and 85 years, there is a $20 \%-25 \%$ reduction in the GFR hence the need for compensatory mechanisms when MA occurs. A common compensatory mechanism in MA is pulmonary hyperventilation, developed to reduce the carbon dioxide load of the blood by stimulating the brain stem respiratory pathway. The presence of respiratory diseases which are more common in the elderly population therefore, makes this population group more vulnerable to and to suffer more deleterious effects of metabolic acidosis [17]. This also contributes to the higher prevalence of MA in the obese population due to the compromised ventilation in them. This obesity related problem is made worse in the supine position due to some degree of closure of the terminal airway, a condition that could impact negatively during surgeries under general anesthesia [18].

The serum albumin is reported to be lower in the elderly [19]. This could be multifactorial in origin, from lower intake, The fluid shift noticed with surgeries of the pancreatic, cardiac and biliary tree further worsens the effects of metabolic acidosis by causing fluid shift, electrolyte and acid-base derangement. The alteration of the pressure gradient between compartments from fluid shift during surgeries has a cause and effect relationship with changes in membrane potential [20]. The restoration of normal acid base balance in acidosis could be partly achieved by the loss of calcium from bone to buffer the excess acid and this results in hypercalcemia. The demineralization of bone could precipitate the formation of kidney stones, fractures, osteolytic lesions and the development of the CKD bone mineral disease (CKD-BMD), a common finding in patients with $\mathrm{CKD}$, and with hyperparathyroidism [21].

Perioperative metabolic acidosis is reported to be a risk factor for poor treatment outcome on the surgical ward more so in the intensive care units due to greater incidence of perioperative comorbidities and complications. Highly dependent surgical units dedicated for advance cardiac and vascular procedures are still very few in Nigeria and many low income countries hence the paucity of literature on the relationship between perioperative biochemical state and treat- 
ment outcome. In our clime, we are not aware of any work on perioperative metabolic acidosis and treatment outcome in this population. We therefore studied perioperative metabolic acidosis in this population and determined its risk factors, relationship with post-operative events and treatment outcome.

\section{Patients and Methods}

This was a cohort retrospective study carried in the "Tristate Heart and vascular Center" a high dependency cardiovascular surgical unit of a tertiary health institution in Ilishan-Remo, South west Nigeria dedicated to managing patients requiring heart and vascular surgical procedures. Case notes and perioperative charts of patients treated in the center from January 2015 to December 2019 were retrieved. Variables obtained from the case files included age, gender, type and cause of cardiovascular disease, duration of disease, previous surgical procedure related to disease, class of surgery, body mass index (BMI), percentage oxygen saturation, pulse rate, blood pressures (BP), heart rate, duration of hospital stay till discharge or death and comorbidities (heart failure, chronic liver disease, chronic kidney disease, stroke or cancer). Also retrieved from the case files were the preoperative, post-operative day $1\left(\mathrm{POD}^{1}\right)$ and post-operative day $28\left(\mathrm{POD}^{28}\right)$, urinalysis, serum electrolyte, urea and creatinine, full blood count (FBC), blood glucose, fasting lipids, arterial blood gases and liver function test results and erythrocyte sedimentation rate (ESR).

Participants less than 16 years or those that had previous surgery within 30 days prior to the index surgery or had repeat surgeries for the same condition and those whose data were missing were excluded.

According to the unit protocol, blood samples for arterial blood gases, serum electrolytes, albumin, fasting lipids, full blood count (FBC) and blood glucose were taken before incision, with participants awake, and samples for serum electrolytes, FBC and blood glucose were taken 24 hours post-surgery, POD 7 and on POD 28 whether patient was on admission or had been discharged, as unit protocol demanded a review of all patients, with laboratory tests on POD 28. Where infection occurred, type and site were documented. The types of infusions and inotropes used were documented, and including the charted urinary output.

For the purpose of this study, the following definitions were used:

Metabolic acidosis-serum bicarbonate $\left(\mathrm{HCO}_{3}\right)<20 \mathrm{mmol} / \mathrm{l}[1]$.

Kidney dysfunction-GFR $<60 \mathrm{ml} / \mathrm{min}$ [22].

Hypertension-BP $\geq 140 / 90 \mathrm{mmHg}$ [23].

Diabetes-FBS $>7.0 \mathrm{mmol}[24]$.

Anemia-packed cell volume (PCV) -30\% [25].

Standard base excess $(\mathrm{SBE})-<-2 \mathrm{mEq}^{-1}[11]$.

Anion gap (AN) $-\mathrm{Na}+\mathrm{K}-\mathrm{Cl}-\mathrm{HCO}_{3}[11]$.

Data obtained were analyzed using IBM SPSS software version 22 (IBM Inc, NY, USA). Continuous variables were expressed as means with standard deviations and compared using Student t-test. Categorical variables were expressed as 
frequencies and percentages and compared using Chi square test. Where 3 or more variables were compared, analysis of variance (ANOVA) was used. The level of $\mathrm{P}<0.05$ was considered statistically significant. The research followed the tents of the Declaration of Helsinki. The Ethics Committee of Babcock University approved this study. The institutional ethical committee of Babcock University approved the study (BUHREC558/19, NHREC/24/01/2018).

\section{Results}

Five hundred and thirty six patients had surgeries in the center during the period studied. After applying the exclusion criteria, 342 participants took part in the study and of this, 128 (37.4) had open heart surgery, 101 (29.5) had major vascular surgery and 113 (33.1) had intermediate/minor surgeries. Two hundred and six (60.2\%) were males and $136(39.8 \%)$ were females. The mean age of the participants was $53.2 \pm 143$ years, with males $51.9 \pm 8.4$ and females, $55.1 \pm 4.5$. The demographic and clinical characteristics of the participants is shown in Table 1. A greater proportion of participants, 198 (57.9\%), were 50 years or more. Difficulty with breathing 182 (53.2\%) was the commonest presenting compliant followed by chest pain 171 (50.0\%). Majority of participants had low or normal BMI. One hundred and four (30.4\%) participants had comorbidities, 77 (74.0\%) of these were males with 27 (26.0\%) females.

Table 1. Preoperative sociodemographic and clinical characteristics of the participants.

\begin{tabular}{ccc}
\hline Variables & Frequency & Percentage \\
\hline Gender & 206 & 39.8 \\
Male & 136 & 60.2 \\
Female & & \\
Age, years & 144 & 42.1 \\
$16.0-49.9$ & 198 & 57.9 \\
$\geq 50$ & & \\
$\mathrm{BMI}, \mathrm{kg} / \mathrm{m}^{2}$ & 179 & 52.3 \\
$<25.0$ & 163 & 47.7 \\
$\geq 25.0$ & & \\
$\mathrm{SBP}, \mathrm{mmHg}$ & 147 & 43.0 \\
$<140$ & 195 & 57.0 \\
$>140$ & & 51.8 \\
$\mathrm{DBP}, \mathrm{mmHg}$ & 143 & 58.2 \\
$<90$ & 199 & 30.1 \\
$\geq 90$ & 239 & \\
SPO & & \\
$<95$ & 103 & \\
$>95$ & & \\
\hline
\end{tabular}

$\mathrm{BMI}$-body mass index, $\mathrm{SBP}$ - systolic blood pressure, $\mathrm{DBP}$ - diastolic blood pressure, $\mathrm{SPO}_{2}$ - percentage oxygen saturation. 
At presentation, 70 (20.5\%) participants had metabolic acidosis as against 272 (79.5\%) that had normal serum bicarbonate. On POD $^{1} 136$ (39.8\%) participants had MA compared to 206 (60.2\%) with normobicarbonemia. On POD ${ }^{28}$, forty seven (14.1) had MA compared to 286 (85.9\%) with normal serum bicarbonate, $\mathrm{P}<0.001$.

The mean serum bicarbonate, pre surgery, on $\mathrm{POD}^{1}$ and on $\mathrm{POD}^{28}$ were 19.3 $\pm 2.7 \mathrm{mmol}, 18.1 \pm 2.8 \mathrm{mmol}$ and $21.6 \pm 5.3$ respectively, $\mathrm{P}<0.001$. The laboratory results of participants is shown in Table 2 . The mean eGFR, pre-op, on $\mathrm{POD}^{1}$ and on $\mathrm{POD}^{28}$ were $73.6 \pm 9.7 \mathrm{ml} / \mathrm{min}, 56.4 \pm 17.9 \mathrm{ml} / \mathrm{min}$ and $86.5 \pm 21.8$ $\mathrm{ml} / \mathrm{min}$ respectively, $\mathrm{P}<0.001$. There were significant differences in the mean neutrophil count, platelet count and serum creatinine, at presentation, on POD $^{1}$ and on $\mathrm{POD}^{28}, \mathrm{P}<0.001, \mathrm{P}=0.04$ and $\mathrm{P}<0.001$ respectively. There were no significant difference in the serum albumin and ESR at presentation and on $\mathrm{POD}^{28}$, $\mathrm{P}=0.05$ and $\mathrm{P}=0.9$ respectively.

Table 3 shows that males have the larger proportion of metabolic acidosis at all stages of the study though the proportion of females with MA increased on $\mathrm{POD}^{1}, \mathrm{P}=0.001$. MA was commoner among participants 50 years and upwards compared with those less than 50 years, P, 0.001 As the BMI increased, the proportion of participants with MA increased, the difference was further widen on $\mathrm{POD}^{1}, \mathrm{P}<0.001$. Metabolic acidosis was more common among participants who had open heart surgery and even more so, on $\mathrm{POD}^{1}, \mathrm{P}<0.001$.

Table 2. Laboratory results of the study population.

\begin{tabular}{|c|c|c|c|c|}
\hline Variables & Preoperative & $\mathrm{POD}^{1}$ & $\mathrm{POD}^{28}$ & P-value \\
\hline & $\mathrm{N}=342$ & $\mathrm{~N}=342$ & $\mathrm{~N}=333$ & ANOVA \\
\hline Sodium, mmol & $132.3 \pm 3.5$ & $130.6 \pm 9.4$ & $138.2 \pm 11.6$ & 0.001 \\
\hline Potassium, mmol & $4.2 \pm 1.5$ & $4.8 \pm 1.4$ & $3.8 .2 \pm 1.1$ & 0.03 \\
\hline Chloride, mmol & $102.3 \pm 1.5$ & $111.8 \pm 1.4$ & $99.2 \pm 1.1$ & 0.03 \\
\hline Bicarbonate, $\mathrm{mmol}$ & $19.3 \pm 2.7$ & $18.1 \pm 2.8$ & $21.6 \pm 5.3$ & $<0.001$ \\
\hline Anion gap, mEq/l & $5.2 \pm 1.2$ & $1.4 \pm 0.7$ & $8.9 \pm 4.5$ & $<0.001$ \\
\hline Urea, mmol & $8.8 \pm 2.6$ & $11.3 \pm 3.7$ & $9.0 \pm 3.1$ & 0.001 \\
\hline Creatinine, umol & $114.5 \pm 14.2$ & $143.8 \pm 7.7$ & $99.1 \pm 15.8$ & $<0.001$ \\
\hline GFR, ml/min (CKD-EPI) & $73.6 \pm 9.7$ & $143.8 \pm 7.7$ & $86.5 \pm 21.8$ & $<0.001$ \\
\hline Total WBC, $\times 10^{9} / 1$ & $5.6 \pm 8.3$ & $6.3 \pm 4.9$ & $5.2 \pm 4.1$ & 0.003 \\
\hline Neutrophils, $\times 10^{9} / 1$ & $2.9 \pm 3.3$ & $3.9 \pm 2.7$ & $2.4 \pm 1.8$ & $<0.001$ \\
\hline $\mathrm{PCV}, \%$ & $36.8 \pm 3.6$ & $362 \pm 2.3$ & $37.5 \pm 4.2$ & 0.05 \\
\hline Glucose, mmol & $5.8 \pm 2.1$ & $5.9 \pm 4.1$ & $5.1 \pm 1.9$ & 0.1 \\
\hline Platelets, $\times 10^{9 /} 1$ & $235.8 \pm 12.4$ & $276.4 \pm 32.4$ & $243.6 \pm 5.4$ & 0.04 \\
\hline Albumin, $g / 1$ & $38.7 \pm 5.9$ & & $39.8 \pm 9.4$ & 0.05 \\
\hline $\mathrm{ESR}, \mathrm{mm} / \mathrm{hr}$ & $11.8 \pm 5.2$ & & $11.3 \pm 3.2$ & 0.9 \\
\hline
\end{tabular}

POD—post operative day, GFR - glomerular filtration rate, CKD-EPI—chronic kidney disease epidemiology collaboration, WBC_-white cell count, PCV—-packed cell volume, ESR_erythrocyte sedimentation rate. 
Table 3. Relationship between pre-op metabolic acidosis and participants' characteristics.

\begin{tabular}{|c|c|c|c|c|c|}
\hline Variables & Frequency & Pre-opHCO $\mathrm{H}_{3}$ & $\mathrm{POD}^{1} \mathrm{HCO}_{3}$ & $\mathrm{POD}^{28} \mathrm{HCO}_{3}$ & $\mathrm{P}$-value \\
\hline & $\mathrm{N}=342(\%)$ & $\mathrm{N}=70(\%)$ & $\mathrm{N}=136(\%)$ & $\mathrm{N}=47(\%)$ & ANOVA \\
\hline & & $<20$ & $<20$ & $<20$ & \\
\hline \multicolumn{6}{|l|}{ Gender } \\
\hline Males & $206(60.2 \%)$ & $43(61.4 \%)$ & $72(52.9 \%)$ & $28(66.1 \%)$ & 0.001 \\
\hline Female & $136(39.8 \%)$ & $27(38.3 \%)$ & $64(47.1 \%)$ & $19(33.9 \%)$ & \\
\hline \multicolumn{6}{|l|}{ Age, years } \\
\hline $16-49$ & $144(42.1 \%)$ & $12(17.1 \%)$ & $22(16.2 \%)$ & $18(38.3 \%)$ & $<0.001$ \\
\hline$\geq 50$ & $198(57.9 \%)$ & $58(82.9 \%)$ & $114(83.8 \%)$ & $29(61.7 \%)$ & \\
\hline \multicolumn{6}{|l|}{ BMI, $k g / m^{2}$} \\
\hline$<25.0$ & $179(52.3 \%)$ & $25(35.7 \%)$ & $28(20.6 \%)$ & $20(42.6 \%)$ & $<0.001$ \\
\hline$\geq 25.0$ & $163(47.7 \%)$ & $(64.3 \%)$ & $108(79.4 \%)$ & $27(57.4 \%)$ & \\
\hline \multicolumn{6}{|l|}{$\mathrm{SBP}, \mathrm{mmHg}$} \\
\hline$<140$ & $144(42.1 \%)$ & $31(44.3 \%)$ & $34(25.0 \%)$ & $22(46.8 \%)$ & 0.003 \\
\hline$\geq 140$ & $198(58.9 \%)$ & $39(55.7 \%)$ & $102(75.0 \%)$ & $25(53.2 \%)$ & \\
\hline \multicolumn{6}{|l|}{$\mathrm{DBP}, \mathrm{mmHg}$} \\
\hline$<90$ & $154(45.0 \%)$ & $25(35.7 \%)$ & $63(46.3 \%)$ & $23(48.9 \%)$ & 0.05 \\
\hline$\geq 90$ & $188(55.0 \%)$ & $45(64.3 \%)$ & $73(53.7 \%)$ & $24(51.1 \%)$ & \\
\hline \multicolumn{6}{|l|}{$\mathrm{SPO}_{2}, \%$} \\
\hline$<95$ & $58(17.0 \%)$ & $27(38.6 \%)$ & $49(36.0 \%)$ & $11(23.4 \%)$ & 0.04 \\
\hline$\geq 95$ & $294(83.0 \%)$ & $43(61.4 \%)$ & $87(64.0 \%)$ & $36(76.6 \%)$ & \\
\hline \multicolumn{6}{|l|}{ Surgery } \\
\hline Open heart & $128(37.4 \%)$ & $49(70.0 \%)$ & $97(71.3 \%)$ & $28(59.6 \%)$ & $<0.001$ \\
\hline Vascular & $101(29.5 \%)$ & $18(25.7 \%)$ & $35(25.7 \%)$ & $17(36.2 \%$ & \\
\hline Intermediate & $113(33.1 \%)$ & $3(4.3 \%)$ & $4(3.0 \%)$ & $2(4.2 \%)$ & \\
\hline
\end{tabular}

BMI-body mass index, SBP-systolic blood pressure, DBP-diastolic blood pressure.

One hundred and four participants had comorbidities, 70 (67.3\%) of this, all (100\%) and 47 (45.2\%) of this had metabolic acidosis prior to surgery, on $\mathrm{POD}^{1}$, and on $\mathrm{POD}^{28}$ respectively. One hundred and eighty four (53.8\%) were on antihypertensive drugs and of this, 127 (69.0\%) were receiving diuretic for blood pressure control.

One hundred and twenty nine (37.7\%) participants had kidney dysfunction (KD) prior to surgery as against 213 (62.3\%) who had no kidney dysfunction. Of the participants with preexisting KD, $39(30.2 \%)$ had significant worsening of kidney function within $\mathrm{POD}^{7}$. Of the participants without preexisting $\mathrm{KD}, 49$ (23.0\%) had acute kidney injury (AKI) within the first $\mathrm{POD}^{7}$. Of the eighty eight participants reviewed by the nephrologist, 57 (64.8\%) were males, 31 were females (35.2\%), 35 participants (39.8\%) underwent hemodialysis treatment.

The relationship between preoperative bicarbonate and status of kidney func- 
tion is shown in Table 4. Fourteen (4.1\%) had moderate-to-severe metabolic acidosis, 56 (16.4\%) had mild MA and 272 (79.5\%) had normal serum bicarbonate.

Forty nine (14.3) participants had infections during admission and all (100.0) had metabolic acidosis. Of the $9(2.7 \%)$ deaths, $6(66.7 \%)$ had metabolic acidosis at presentation and all $(100.0 \%)$ had MA at POD ${ }^{1}$. Two $(22.2 \%)$ of the nine death were within the first three POD, 5 (55.6\%) died between $\mathrm{POD}^{3}$ and $\mathrm{POD}^{7}$ while the remaining 2 (22.2\%) died after the POD 7. Six of the 206 (2.9\%) male participants in the study died on admission compared to 3, out of the $136(2.2 \%)$ female participants that died, $\mathrm{P}=0.002$. Two of the $144(1.4 \%)$ participants less than 50 years died during admission compared to 7 of the 198 (3.5\%) participants that were up to 50 years, $\mathrm{P}<0.001$. Comorbidities were more in males. Twenty one percent of all participants but eighty six of the participants with metabolic acidosis had comorbidities, $\mathrm{P}=0.003$. The relationship between the preoperative MA and treatment outcome is showed in Table 5. There was an inverse relationship between serum bicarbonate level and duration of admission. A higher percentage of those that were on admission for up to 28 days had severe MA while a higher percentage of those on admission 3 - 7 days had normal serum bicarbonate. The difference was statistically significant, $\mathrm{P}<0.001$. There was an inverse relationship between the number of comorbidities and the serum bicarbonate. The highest percentage of participants without comorbidity had normal bicarbonate whereas the largest percentage of participants with 3 or more comorbidities had severe MA. The difference was statistically significant, $\mathrm{P}$ $<0.001$.

Multivariate regression analysis in Table 6 showed advancing age (OR-7.82, CI-5.63 - 8.47, P = 0.02), longer duration of hospital stay (OR-1.23, CI-1.14 $2.91, \mathrm{P}=0.001$ ), type of surgery (OR-0.048, CI-0.012 - 0.153, P $<0.001$ ), Comorbidities (OR-2.62, CI-1.44 - 7.67, P $<0.001$ ), elevated SBP (OR-9.63, CI-7.88 $10.34, \mathrm{P}=0.02$ ), and low eGFR (OR-4.35, CI-2.74 - 5.85, $\mathrm{P}=0.01$ ) were predictors of perioperative metabolic acidosis.

Table 4. Relationship between Preoperative serum Bicarbonate and status of kidney function.

\begin{tabular}{cccccc}
\hline Variables & All & Bicarbonate & Bicarbonate & Bicarbonate & P-value \\
\hline & Participants & $<18 \mathrm{mmol}$ & $18-19.9 .0$ & $\geq 20 \mathrm{mmol}$ & \\
& $\mathrm{N}=342(\%)$ & $\mathrm{N}=14(\%)$ & $\mathrm{N}=56(\%)$ & $\mathrm{N}=272(\%)$ & \\
GFR.ml/min (CKD-EPI) & & & & & \\
$\geq 90.0$ & $65(19.0 \%)$ & $0(0.0 \%)$ & $7(12.5 \%)$ & $58(21.3 \%)$ & $<0.001$ \\
$60-89.9$ & $148(43.3 \%)$ & $0(0.0 \%)$ & $48(85.7 \%)$ & $100(36.8 \%)$ & \\
$45-59.9$ & $114(33.3 \%)$ & $1(7.1 \%)$ & $1(1.8 \%)$ & $112(41.2 \%)$ & \\
$30-44.9$ & $12(3.5 \%)$ & $10(71.4 \%)$ & $0(0.0 \%)$ & $2(0.7 \%)$ & \\
$15-29.9$ & $3(0.9 \%)$ & $3(21.5 \%)$ & $0(0.0 \%)$ & $0(0.0 \%)$ & \\
\hline
\end{tabular}

GFR—glomerular filtration rate, CKD-EPI-chronic kidney disease epidemiology collaboration. 
Table 5. Relationship between Preoperative metabolic acidosis and treatment outcome.

\begin{tabular}{cccccc}
\hline Variables & Frequency & Bicarbonate & Bicarbonate & Bicarbonate & P-value \\
\hline & $\mathrm{N}=342(\%)$ & $<18.0$ & $18.0-19.9$ & $>20.0$ & ANOVA \\
Days on admission & & $\mathrm{N}=14(\%)$ & $\mathrm{N}=56(\%)$ & $\mathrm{N}=272(\%)$ & \\
$3-7$ & $127(37.2 \%)$ & $0(0.0 \%)$ & $16(28.6 \%)$ & $111(40.8 \%)$ & $<0.001$ \\
$8-14$ & $128(37.5 \%)$ & $2(20.0 \%)$ & $8(14.3 \%)$ & $118(43.4 \%)$ & \\
$15-28$ & $61(17.8 \%)$ & $4(58.6 \%)$ & $18(32.1 \%)$ & $39(14.3 \%)$ & \\
$>28$ & $26(10.5 \%)$ & $8(21.4 \%)$ & $14(25.0 \%)$ & $4(1.5 \%)$ & \\
Comorbidities & & & & & \\
None & $218(63.8 \%)$ & $2(14.3 \%)$ & $15(26.8 \%)$ & $201(73.9 \%)$ & $<0.001$ \\
1 & $74(21.6 \%)$ & $6(42.8 \%)$ & $24(42.9 \%)$ & $44(16.2 \%)$ & \\
2 & $42(12.3 \%)$ & $4(28.6 \%)$ & $11(19.6 \%)$ & $27(9.9 \%)$ & \\
3 & $8(2.3 \%)$ & $2(14.3 \%)$ & $6(10.7 \%)$ & $0(0.0 \%)$ & \\
Yes & $35(10.2 \%)$ & $6(42.9 \%)$ & $2(3.6 \%)$ & $27(9.9 \%)$ & \\
No & & & & & \\
\hline
\end{tabular}

*-fisher's exact test.

Table 6. Multivariate regression analysis.

\begin{tabular}{cccc}
\hline Variables & OR & $95 \%$ CI & P-value \\
\hline Sex & 1.32 & $1.26-1.46$ & 0.06 \\
Age & 7.82 & $5.63-8.47$ & 0.02 \\
Surgery type & 0.048 & $0.012-0.153$ & $<0.001$ \\
Comorbidities & 2.62 & $1.44-7.67$ & $<0.001$ \\
Length of admission & 1.23 & $1.14-2.91$ & 0.001 \\
BMI & 5.56 & $5.27-7.11$ & 0.05 \\
SBP & 9.63 & $7.88-10.34$ & 0.02 \\
DBP & 2.92 & $2.74-2.99$ & 0.05 \\
SPO2 & 0.03 & $0.02-0.41$ & 0.06 \\
eGFR & 4.35 & $2.74-5.85$ & 0.01 \\
\hline
\end{tabular}

OR—odd ratio, $\mathrm{CI} —$ confidence interval, BMI-body mass index, SBP—systolic blood pressure, DBP-diastolic blood pressure, $\mathrm{SPO}_{2}$ - percentage oxygen saturation, eGFR - estimated glomerular filtration rate.

\section{Discussion}

We found in our assessment of perioperative metabolic acidosis in a high dependency heart and vascular center, a incidence of $20.5 \%$ of preoperative MA. This rose to $39.7 \%$ on $\mathrm{POD}^{1}$ and decreased to $14.1 \%$ on $\mathrm{POD}^{28}$. This preoperative prevalence is similar to findings by Lawton et al. who reported a prevalence of $17 \%$ of metabolic acidosis prior to induction which rose to $42 \% 12 \mathrm{hrs}$ after 
surgery. Metabolic acidosis on the surgical intensive could arise from fasting and fluid administration. The rising prevalence of MA between induction and $\mathrm{POD}^{1}$ found in our study and that of Lawton et al. could be attributable to the stress of surgery leading to increased release of catecholamine and diversion of the glycolytic pathway from pyruvate to lactate production [11]. The rising incidence of acidosis can also result from reduced blood flow to the vital organs consequent upon intra operative blood loss, redistribution of fluid and reduced adenosine triphosphate (ATP) secondary to ongoing inflammation [20].

The male dominance in our study is also reported by Jouldi et al. in a series on post-operative MA [26]. In Nigeria and many Sub Sahara African countries, cultural, educational and economic bias against women have impacted negatively on their health seeking capacity [27]. Though MA was more prevalent in males, it is worth noting that the incidence of MA increased in women at $\mathrm{POD}^{1}$. Casimir et al. reported a higher incidence of MA in women in acute inflammatory conditions associated with a more profound peripheral polymorphnuclear cell infiltration and reduction in the $\mathrm{PH}$ of the blood. The authors concluded that the relative health survival advantage of women in acute inflammatory conditions was secondary to their ability to mount effective inflammatory response during infections and trauma [28].

In fed states, females convert most of the free fatty acids to triglycerides and then to storage tissues, and during fasting and exercise, their energy source are drawn mostly from remaining fatty acids. Males on the other hand oxidizes free fatty acids, therefore, during starvation and exercise, they draw their energy from carbohydrate stores. Males by having less energy reserve are therefore prone to catabolic degradation of protein stores and ketosis in prolonged fasting states often experience in many perioperative patients [28]. The higher prevalence and severity of cardiovascular diseases in males could also be contributory to the higher incidence of MA in males. Miller et al. reported that males manifest a heightened response to adrenergic stimulation and in the presence of adrenergic inhibition, males show lesser responsiveness [29]. In addition, the protective role of estrogens in altering serum lipids and mitigating the release of pro-inflammatory cytokines that follows endothelial injury also contribute to the relative survival advantage of women in acute inflammatory states [12].

We found a direct relationship between perioperative MA and age, this mirrors findings by Frassetto et al. [30] who found that background MA, particularly if arising from chronic kidney disease, was a risk factor for accelerated aging. Zampieri et al. found a positive correlation between cytokines like IL-1 $\beta$, IL-6, TNF- $\alpha$ and hydrogen peroxide $\left(\mathrm{H}_{2} \mathrm{O}_{2}\right)$ The release of anorexic cytokines, oxidative stress and a tilting of the body metabolic pathway in favor of the catabolic cascade is associated with chronic tissue degradation and apoptosis common in the aging process [30]. Reduced tubular ammonia generation and acid secretion from the Type A intercalated cells are also contributory to the higher incidence of MA with aging [31]. 
There was an inverse relationship between the body mass index (BMI) and serum bicarbonate, a negative correlate of MA. This mirrors findings by Souto et al. who reported that obesity was a risk factor for MA [32]. We also found a surge in the incidence of $\mathrm{MA}$ on $\mathrm{POD}^{1}$ and this is similar to findings by Al-Mulhim et al. who reported a high postoperative MA in many surgical procedures involving major organs of the body [33]. Obesity could be associated with a compromised ventilation especially during surgeries. The combined effect of hypoventilation from relative closure of the terminal airways on the operative table (in the supine position) seen with general anesthesia (GA), poor positioning, physiologic response to pneumothorax and pneumoperitoneum all contribute to worsening of metabolic acidosis intraoperative and early postoperative periods [18].

Metabolic acidosis was more common in the hypertensive population than in the normotensive participants. Taylor et al. [34] in a nationwide nutritional examination and survey, found in salt sensitive hypertensive in the United states, a direct relationship between elevated blood pressure on one hand and increased renal tubular acid secretion, increased endogenous acid generation and metabolic acidosis. The authors concluded that the positive relationship between MA and hypertension was more dependent on organic acid generation than on net renal acid loss. Metabolic acidosis act a trigger for the release of catecholamine, endothelin-1, thromboxane A and aldosterone hence its role in the development of increased left ventricular mass and abnormal geometry [35].

Our study found oxygen desaturation prior to induction more prevalent than at $\mathrm{POD}^{1}$. General anesthesia and mechanical ventilation impair pulmonary function resulting in low tissue oxygenation (low $\mathrm{SPO}_{2}$ ) [18]. It is therefore expected that the $\mathrm{SPO}_{2}$ would be positively related to the bicarbonate concentration both in the pre and postoperative periods [36]. We infer that this unexpected finding is most likely due to the concurrent oxygen therapy that most of the participants were receiving 24 hours after surgery particularly those in the ICU.

Metabolic acidosis was most prevalent amongst participants that had open heart surgery than vascular and intermediate surgeries. Filipescus et al. found high incidence of MA after open heart surgeries [37]. The increase in the incidence of MA from induction to $\mathrm{POD}^{1}$ is commonly associated with hyperchloremia (more than hyperlactatemia), increase anion gap and decrease PH. Metabolic acidosis was commonest on $\mathrm{POD}^{28}$ amongst those that had vascular surgery. Choudhury et al. found a high incidence of MA in the immediate postoperative period and concluded that respiratory acidosis tend to play a more causative role than metabolic acidosis, with more involvement of the renal compensatory mechanisms [38]. Cassese et al. reported a high rate of vascular restenosis in cardiopulmonary bypass surgeries and listed diabetes, small vessel disease, chronic kidney disease as risk factors for restenosis [39]. The author also found a direct relationship between the restenosis and the number of vessels involved in disease process. Restenosis could also be accelerated by ongoing inflammation mediating vasoconstriction, platelet aggregation and release of trans- 
forming growth factor $\beta$ (TGF $\beta$ ) and fibroblast growth factor with suppression of nitric oxide $(\mathrm{NO})$ release.

The prevalence of preoperative kidney dysfunction among the participants was $37.7 \%$ The incidence and severity of MA increased with the stages of KD and this mirrors findings by Stancu et al. [40] CKD is associated with reduced tubular ammonia excretion and reduced tubular (proximal tubules and collecting ducts) ammonia absorption [28]. Though it is known that metabolic acidosis increases the risk of mortality in CKD, it is also reported that vascular calcification is inhibited by chronic acidosis as a result of suppressed collangen synthesis, coupled with the fact that arteriolosclerosis is inhibited from concurrent osteoblast inhibition in MA [41].

We found postoperative infection as a risk factor for death in our series and a predominance of males among the dead. The ability of females to mount effective inflammatory response in sepsis, thereby having a survival advantage over males, has been attributed to this response characterized low $\mathrm{PH}$ and polymorphonuclear cell infiltration. The relative higher energy store of the X-chromosome, anti-inflammatory actions of estrogens, anti angiogenic actions of estrogens and a greater tendency to lipid peroxidation by females gives them a survival advantage [42]. We found a positive association between MA and death similar to findings by Gunnerson et al. [41] Comorbid conditions, particularly $\mathrm{CKD}$, were associated with higher incidence of MA in the study. Apart of the known fact that males have more severe forms hypertension and cardiovascular disease as possible risk factor or death in them, we also infer that the higher death rate among males in the study could also be explained on the basis of other comorbidities, like CKD. The findings of lower GFR, higher parathyroid hormone and higher cholesterol associated with MA, by Capusa et al. further buttress this association [43]. The kidney disease improving global outcome (KDIGO) Clinical Practice Guideline for the evaluation and management of CKD recommended bicarbonate supplementation when $\mathrm{HCO}_{3}$ is less than $22 \mathrm{mmol}$ [44]. Though $37.7 \%$ of participants had KD prior to induction, we found a rising percentage of participants with MA associated with decreasing renal function that necessitated the nephrologist review in about $25.7 \%$ of participants, out of which $10 \%$ had hemodialysis. Raji et al. [45] reported a $22.5 \%$ incidence rate of perioperative $\mathrm{AKI}$ at $\mathrm{POD}^{7}$, similar to our finding. Kidney disease are commonly associated with cardiovascular disorders with each worsening the outcome of the other when they occur together [21].

Although MA was most prevalent on $\mathrm{POD}^{1}$, we found a direct relationship between the length of admission and the severity of MA. Metabolic acidosis in the perioperative period could be pointers to ongoing infections as was found in this study. The additive effect of infections to the disease burden often prolong the wound healing time as collagen formation could become dysregulated necessitating longer treatment time [41]. Metabolic acidosis being a proinflammatory condition, it expect that the release of anorexic cytokines could disrupt the ana- 
bolic state that is needed for effective wound healing thereby delay it but Tsuji et al. reported that hypercapnia, not acidosis cause stimulation of cell proliferation thereby accelerating wound healing [46].

On multivariate logistic regression, advancing age ( $\geq 50 \mathrm{yrs}$ ), duration of admission $\left(<\mathrm{POD}^{2}\right)$, surgery type (open heart surgery), systolic BP $(\geq 140)$ independently predicted metabolic acidosis.

There were some limitations encountered in this study. Data on base excess were not available for many intermediate grade surgeries so was not used in defining MA. Though Hartman solution was used in most cases, data on the use of other solution were incomplete. The effect of perioperative blood transfusion was not determined in the study. Urine osmolality was also not assessed.

\section{Conclusion}

Metabolic acidosis is common in perioperative patients as we found incidence of $20.4 \%, 39.8 \%$ and $14.1 \%$ just prior to induction, on $\mathrm{POD}^{1}$ and $\mathrm{POD}^{28}$ respectively. The risk factors for MA were advanced age ( $\geq 50 \mathrm{yrs}$ ), comorbidities (CKD), type of surgery (open heart surgery), systolic blood pressure $(\geq 140 \mathrm{mmHg})$ and eGFR $<60 \mathrm{~min} / \mathrm{ml}$. The presence of metabolic acidosis increased the risk for prolonged hospital stay, decline in kidney function and death. Worsening of kidney function from baseline was more common among participants with preexisting kidney dysfunction than in those without preexisting kidney disease. There is therefore need to optimize the acid base balance in patients with advanced age, $\mathrm{CKD}$ and comorbidities to achieve more favorable outcome in patients undergoing cardiovascular surgeries. More multicenter and multi-racial studies are needed to standardize management protocols.

\section{Acknowledgements}

We express our profound gratitude to the nurses, technical, administrative staff of Tristate Heart and Vascular Center, Ilishan-Remo, Nigeria for their contribution to the success of this study.

\section{Conflicts of Interest}

The authors declare no conflicts of interest regarding the publication of this paper.

\section{References}

[1] Sanchez-Diaz, J.S., Martinez-Rodriguez, E.A., Mendez-Rubio, L.P., et al. (2016) Acid-Base Balance. Update. Henderson-Hasselbalch Theory. Medicina Interna de México, 32, 646-660.

[2] Farhangi, M.A., Nikniaz, L. and Nikniazn, Z. (2019) Higher Dietary Acid Load Potentially Increases Serum Triglyceride and Obesity Prevalence in Adults: An Updated Systematic Review and Meta-Analysis. PLOS ONE, 14, e0216547. https://doi.org/10.1371/journal.pone.0216547

[3] Taylor, E.N. and Curhan, G.C. (2006) Body Size and 24-Hour Urine Composition. American Journal of Kidney Diseases, 48, 905-915. 
https://doi.org/10.1053/j.ajkd.2006.09.004

[4] Akter, S., Eguchi, M., Kurotani, K., Kochi, T., Pham, N.M., Ito, R., et al. (2015) High Dietary Acid Load Is Associated with Increased Prevalence of Hypertension: The Furukawa Nutrition and Health Study. Nutrition, 31, 298-303.

https://doi.org/10.1016/j.nut.2014.07.007

[5] van den Berg, E., Engberink, M.F., Brink, E.J., van Baak, M.A., Joosten, M.M., Gans, R.O.B., et al. (2012) Dietary Acid Load and Metabolic Acidosis in Renal Transplant Recipients. Clinical Journal of the American Society of Nephrology, 7, 1811-1818. https://doi.org/10.2215/CJN.04590512

[6] Schiraldi, F., Esposito, G. and Ruggiero, E.G. (2006) Metabolic Acidosis. In: Gullo, A., Ed., Anaesthesia, Pain, Intensive Care and Emergency Medicine A.P.I.C.E., Springer, Milano, 455-462. https://doi.org/10.1007/88-470-0407-1 39

[7] Zampieri, F.G., Kellum, J.A., Park, M., Ranzani, O.T., Barberiro, H.V., de Souza, H.P., et al. (2014) Relationship between Acid-Base Status and Inflammation in the Critically Ill. Critical Care, 18, Article No. R154. https://doi.org/10.1186/cc13993

[8] Berkemeyer, S. (2009) Acid-Base Balance and Weight Gain: Are There Crucial Links via Protein and Organic Acids in Understanding Obesity? Medical Hypotheses, 73, 347-356. https://doi.org/10.1016/j.mehy.2008.09.059

[9] Angus, D., Linde-Zwirbe, W., Lidicker, J., Clermont, G., Carcillo, J. and Pinsky, M. (2001) Epidemiology of Severe Sepsis in the United States: Analysis of the Incidence, Outcome, and Cost of Care. Critical Care Medicine, 29, 1303-1310. https://doi.org/10.1097/00003246-200107000-00002

[10] Story, D.A., Poustie, S. and Bellomo, R. (2001) Comparison of Three Methods to Estimate Plasma Bicrbonate in Critically Ill Patients: Henderson-Hasselbalch, Enzymatic, and Strong-Ion-Gap. Anaesthesia and Intensive Care, 29, 585-590. https://doi.org/10.1177/0310057X0102900603

[11] Lawton, T.O., Quinn, A. and Fletcher, S.J. (2019) Perioperative Metabolic Acidosis: The Bradford Anaesthetic Department Acidosis Study. Journal of the Intensive Care Society, 20, 11-17. https://doi.org/10.1177/1751143718772792

[12] Dombrovskly, V., Martin, A., Suncerram, J. and Paz, H. (2005) Facing the Challenge: Decreasing Case Fatality Rats in Severe Sepsis Despite Increasing Hospitalizations. Critical Care Medicine, 33, 2555-2562. https://doi.org/10.1097/01.CCM.0000186748.64438.7B

[13] Sanusi, A.A., Arogundade, F.A., Famurewa, O.C., Akintomide, A.O., Soyinka, F.O., et al. (2009) Relationship of Ultrasonographically Determined Kidney Volume with Measured GFR, Calculated Creatinine Clearance and Other Parameters in Chronic Kidney Disease (CKD). Nephrology Dialysis Transplantation, 24, 1690-1694. https://doi.org/10.1093/ndt/gfp055

[14] Powers, J.S., Buchowski, M., Wang, L., Powers, J.S. and Otoo-Boameh, A. (2012) Total Body Water in Elderly Adults-Assessing Hydration Status by Bioelectrical Impedance Analysis versus Urine Osmolality. Journal of the American Geriatrics Society, 60, 388-390. https://doi.org/10.1111/j.1532-5415.2011.03814.x

[15] Martin, J., Burnier, M. and Lu, H. (2018) Approach to the Syndrome of Inappropriate Antidiuretic Hormone Secretion (SIADH). Revue Médicale Suisse, 14, 2116 2120.

[16] Su, H.A., Hsieh, H.Y., Lee, C.-T., Liao, S.-C., Chu, C.-H. and Wu, C.-H. (2019) Reference Ranges for Ultrasonographic Renal Dimensions as Functions of Age and Body Indices: A Retrospective Observational Study in Taiwan. PLoS ONE, 14, e0224785.https://doi.org/10.1371/journal.pone.0224785 
[17] Hussain-Syed, F., Slutsky, A.S. and Ronco, C. (2016) Lung-Kidney Cross-Talk in the Critically Ill Patient. American Journal of Respiratory and Critical Care Medicine, 194, 402-414. https://doi.org/10.1164/rccm.201602-0420CP

[18] Chau, E.H.L., Mokhlesi, B. and Chung, F. (2013) Obesity Hypoventilation Syndrome and Anesthesia. Sleep Medicine Clinics, 8, 135-147.

https://doi.org/10.1016/j.jsmc.2012.11.006

[19] Cabrerizo, S., Cuadras, D., Gomez-Busto, F., Artaza-Artabe, I., Marin-Ciancas, F. and Malafarina, V. (2015) Serum Albumin Health in Older People: Review and Meta-Analysis. Maturitas, 81, 17-27. https://doi.org/10.1016/j.maturitas.2015.02.009

[20] Voldy, A.N. and Brandstrup, B. (2016) Fluid Therapy in the Perioperative Setting-A Clinical Review. Journal of Intensive Care, 4, Artcle No. 27.

https://doi.org/10.1186/s40560-016-0154-3

[21] Sanusi, A.A., Arogundade, F.A., Oladigbo, M., Ogini, L.M. and Akinsola, A. (2010) Prevalence and Pattern of Renal Bone Disease in End Stage Renal Disease Patients in Ile-Ife, Nigeria. West African Journal of Medicine, 29, 75-80. https://doi.org/10.4314/wajm.v29i2.68201

[22] National Kidney Foundation (2012) KDOQI Clinical Practice Guideline for Diabetes and CKD: 2012 Update. American Journal of Kidney Diseases, 60, 850-886. https://doi.org/10.1053/j.ajkd.2012.07.005

[23] Agarwal, J., Flynn, J., Pogue, V., Rahman, M., Reisin, E. and Weir, M. (2014) Assessment and Management of Hypertension in Patients on Dialysis. Journal of the American Society of Nephrology, 25, 1630-1646.

https://doi.org/10.1681/ASN.2013060601

[24] American Diabetes Association (2020) Classification and Diagnosis of Diabetes: Standards of Medical Care in Diabetes-2020. Diabetes Care, 43, S205-S206. https://doi.org/10.2337/dc20-Sdis

[25] Cappellini, M.D. and Mota, I. (2015) Anemia in Clinical Practice-Definition and Classification: Does Hemoglobin Change With Aging? Seminars in Hematology, 52, 261-269. https://doi.org/10.1053/j.seminhematol.2015.07.006

[26] Joudi, M., Fathi, M., Soltani, G. and Izanloo, A. (2014) Factors Affecting on Serum Lactate After Cardiac Surgery. Anesthesiology and Pain Medicine, 4, e18514. https://doi.org/10.5812/aapm.18514

[27] Ulasi, I. (2008) Gender Bias in Access to Healthcare in Nigeria: A Study of End Stage Renal Disease. Tropical Doctor, 38, 50-52. https://doi.org/10.1258/td.2007.060160

[28] Casimir, G., Lefevre, N., Corazza, F., Duchateau, J. and Chamekh, M. (2018) The Acid-Base Balance and Gender in Inflammation: A Mini Review. Frontiers in Immunology, 9, 475. https://doi.org/10.3389/fimmu.2018.00475

[29] Miller, J.A., Chernet, D.Z., Duncan, J.A., Lai, V., Burns, K.D., Kennedy, C.R.J., et al. (2006) Gender Difference in the Renal Responses to Renin Angiotensin System Blockage. Journal of the American Society of Nephrology, 17, 2554-2560. https://doi.org/10.1681/ASN.2005101095

[30] Frassetto, L.A., Sebastian, A. and DuBose, T.D. (2020) How Metabolic Acidosis and Kidney Disease May Accelerate the Aging Process. European Journal of Clinical Nutrition, 74, 27-32. https://doi.org/10.1038/s41430-020-0693-5

[31] Hage, V., Villian, C., Pelletier, S., Lavillie, M., Drai, J. and Fourque, D. (2019) Bicarbonate Supplement Restores Urinary Klotho Excretion in Chronic Kidney Disease: A Pilot Study. Journal of Renal Nutrition, 29, 285-288.

https://doi.org/10.1053/j.jrn.2018.11.001 
[32] Souto, G., Donapetry, C., Calvino, J. and Adeva, M.M. (2011) Metabolic AcidosisInduced Insulin Resistance and Cardiovascular Risk. Metabolic Syndrome and Related Disorders, 9, 247-253. https://doi.org/10.1089/met.2010.0108

[33] Al-Mulhim, A.S., Al-Hussaini, H.A., Al-Jalal, B.A., Al-Moagal, R.O. and Al-Najjar, S.A. (2014) Obesity Disease and Surgery. International Journal of Chronic Diseases, 2014, Article ID: 652341. https://doi.org/10.1155/2014/652341

[34] Akter, S., Eguchi, M., Kuwahara, K., Kochi, T., Ito, R., Kurotani, K., et al. (2016) High Dietary Acid Load Is Associated with Insulin Resistance: The Furukawa Nutrition and Health Study. Clinical Nutrition, 35, 453-459. https://doi.org/10.1016/j.clnu.2015.03.008

[35] Taylor, E.N., Forman, J.P. and Farwell, W.R. (2007) Serum Anion Gap and Blood Pressure in the National Health and Nutritional Examination Survey. Hypertension, 50, 320-324. https://doi.org/10.1161/HYPERTENSIONAHA.107.092643

[36] Amodu, A. and Abramowitz, M.K. (2013) Dietary Acid, Age, and Serum Bicarbonate Levels among Adults in the United States. Clinical Journal of the American Society of Nephrology, 8, 2034-2042. https://doi.org/10.2215/CJN.03600413

[37] Filipescu, D., Railleanu, I., Luchian, M., Andrei, M. and Tulbure, D. (2006) Hyperchloremic Metabolic Acidosis after Cardiac Surgery. Critical Care, 10, Article No. P200. https://doi.org/10.1186/cc4547

[38] Choudhury, M. (2017) Management of Vascular Surgery Patients: A Brief Review. Clinics in Surgery, 2, Article No. 1584.

[39] Cassese, S., Bryne, R.A., Tada, T., Pinieck, S., Joner, M., Ibrahim, T., et al. (2014) Incidence and Predictors of Restenosis after Coronary Stenting in 10004 Patients with Surveillance Angiography. Heart, 100, 153-159.

https://doi.org/10.1136/heartjnl-2013-304933

[40] Stancu, S., Mircescu, G., Mocanu, A., Capusa, C. and Stefan, G. (2018) Metabolic Acidosis of Chronic Kidney Disease and Cardiovascular Disorders. Maedica (Buchar), 13, 267-272.

[41] Gunnerson, K.J., Saul, M., He, S. and Kellum, J.A. (2006) Lactate versus Non-Lactate Metabolic Acidosis: A Retrospective Outcome Evaluation of Critically Ill Patients. Critical Care; 10, Article No. R22. https://doi.org/10.1186/cc3987

[42] Papathanassoglou, E., Middleton, N., Benbenishty, J., Williams, G., Christofi, M. and Hegadoren, K. (2017) Systematic Review of Sex-Dependant Outcomes in Sepsis. Nursing in Critical Care, 22, 284-292. https://doi.org/10.1111/nicc.12280

[43] Capusa, C., Stefan, G., Stancu, S., Lipman, M., Tsur, L.D. and Mircescu, G. (2017) Metabolic Acidosis of Chronic Kidney Disease and Subclinical Cardiovascular Disease Markers. Friend of Foe? Medicine, 98, e8802. https://doi.org/10.1097/MD.0000000000008802

[44] Kidney Disease: Improving Global Outcomes and CKD Work Group (2013) KDIGO 2012 Clinical Practice Guideline for the Evaluation and Management of Chronic Kidney Disease. Kidney International Supplements, 3, 1-150.

[45] Raji, Y.M., Ajayi, S.O., Ademola, A.F., Lawal, T.A., Ayandipo, O.O., Adigun, T., et al. (2018) Acute Kidney Injury among Adult Patients Undergoing Major Surgery in a Tertiary Hospital in Nigeria. Clinical Kidney Journal, 11, 443-449. https://doi.org/10.1093/ckj/sfx144

[46] Tsuji, T., Aoshiba, K., Itoh, M., Nakamura, H. and Yamaguchi, K. (2013) Hypercapnea Accelerates Wound Healing in Endothelial Cell Monolayers Exposed to Hypoxia. The Open Respiratory Medicine Journal, 7, 6-12. https://doi.org/10.2174/1874306401307010006 